\title{
Treatment-Free Remission: the New Goal in CML Therapy
}

\author{
Ehab Atallah ${ }^{1}\left[\right.$. Kendra Sweet ${ }^{2}$ \\ Accepted: 15 September 2021 / Published online: 7 October 2021 \\ (c) The Author(s), under exclusive licence to Springer Science+Business Media, LLC, part of Springer Nature 2021
}

\begin{abstract}
Purpose of Review Treatment-free remission (TFR) is considered one of the main goals of therapy in patients with CML. Our goal in this paper is to review the current data on TFR, and discuss future directions.

Recent Findings Multiple studies have demonstrated that attempting a treatment-free remission is safe and effective in a select group of patients. More recent data suggested that undetectable BCR-ABL1 by digital PCR prior to discontinuation is highly predictive of successful TFR. However, some patients have a successful TFR with no evidence of clinical disease despite persistent detectable BCR-ABL1. Some recent studies have shed some more light on possible mechanisms for this phenomena. Some possible mechanisms include immune mechanism, BCR-ABL1 detected in the lymphoid component only, or stem cell exhaustion.

Summary TFR should be discussed with patients with CML. Patients who achieve a sustained deep molecular response may be eligible to attempt TFR, however, setting expectations that overall only $20 \%$ of patients with newly diagnosed CML will achieve a successful TFR. The importance of compliance to treatment early on cannot be overemphasized. Further studies using other drugs to get patients to a deeper remission in order to be eligible for TFR attempt, or attempting a second TFR in patients who had disease recurrence after first TFR attempt, are currently underway.
\end{abstract}

Keywords CML · Tyrosine kinase inhibitors · Treatment-free remission · Digital PCR

\section{Introduction}

Chronic myeloid leukemia (CML) is a myeloproliferative neoplasm which is characterized by the presence of the Philadelphia chromosome. The Philadelphia chromosome results from a reciprocal translocation, $\mathrm{t}(9 ; 22)(\mathrm{q} 34 ; \mathrm{q} 11.2)$, and causes the fusion of the BCR and ABL1 genes, forming a new $B C R-A B L 1$ oncogene [1]. In the early 2000 s, the management of CML was revolutionized by the development of the first ABL1 tyrosine kinase inhibitor (TKI), imatinib [2]. Since that time, four other TKIs, dasatinib, nilotinib, bosutinib, and ponatinib, have gained regulatory approval.

This article is part of Topical Collection on Chronic Myeloid Leukemias

Ehab Atallah

eatallah@mcw.edu

1 Division of Hematology and Oncology, Medical College of Wisconsin, 9200 W. Wisconsin Ave, Milwaukee, WI 53226, USA

2 Moffitt Cancer Center, 12902 USF Magnolia Drive, Tampa, FL 33612, USA
All of these drugs have the potential to induce deep and durable molecular responses in patients with chronic phase (CP) CML, leading those who treat this disease to shift focus toward long-term expectations and quality of life rather than simply short-term disease control [3-6]

\section{Considerations for TKI Cessation}

During the first decade after TKIs were initially approved, the goal of therapy was to improve progression-free and overall survival. However, over time, the goals of therapy have evolved given the durability of deep molecular responses produced by TKIs. With a life expectancy approaching that of the general population, the focus for patients living with CP-CML has shifted toward quality of life and cost savings. Sharf and colleagues surveyed over $1000 \mathrm{CML}$ patients to ask the primary reasons they would consider stopping therapy. They found that $51 \%$ would consider stopping in order to eradicate current TKI side effects, $42 \%$ were worried about the potential for long-term side effects, 37\% did not want to take a medication every day, 
$17 \%$ considered it because their physician recommended enrolling on a TKI discontinuation clinical trial, and $10 \%$ were interested in reducing costs [7]. Another study presented by Goldberg et al. surveyed 210 patients and found the most common motivators for attempting TKI cessation were the possibility of decreasing side effects in $40 \%$, a desire to decrease cost in $30 \%$, and the inconvenience of remaining on daily therapy in $26 \%$ [8].

Although there are many compelling reasons for eligible patients to attempt TKI discontinuation, many patients have expressed hesitancy over this when the opportunity presents itself [9]. The study by Goldberg found that only $42 \%$ of patients were willing to stop treatment, and the primary reasons for remaining on TKIs included concerns regarding disease recurrence (58\%) and absence of current therapyrelated side effects (17\%) [8]. Another study reported that $57 \%$ of patients had a fear of disease recurrence after stopping treatment, while $16 \%$ felt they did not have enough information related to TKI cessation and $12 \%$ were concerned about TKI withdrawal syndrome [7].

Nevertheless, with a growing body of data regarding long-term cardiovascular toxicities from TKIs, along with objective evidence that TKI cessation results in clinically meaningful improvement in patient-reported outcomes such as fatigue, pain, sleep, and diarrhea, and finally clear documentation of significant cost savings associated with stopping therapy, the goal of achieving a treatment-free remission is becoming ever more desirable $[10 \bullet \bullet, 11,12 \bullet \bullet]$.

\section{Who Is Eligible to Attempt Treatment-Free Remission (TFR)?}

Attempting TFR is currently recommended for patients who have been on TKI for at least 3 years, have a sustained deep molecular response (sDMR) for at least 2 years, are in chronic phase CML, and have no evidence of an ABL kinase domain mutation [13, 14]. These recommendations are based on the inclusion criteria used in multiple large TFR studies. As will be discussed later, a longer duration of TKI therapy is associated with a better chance of TFR. Realistically, only $10-20 \%$ of patients would achieve MR4 or MR4.5 by 2 years [3-5], and most patients will be on a TKI for at least 3 years prior to being eligible to attempt TFR. The definition of sDMR has also varied in studies. In the initial STIM1 [15] and TWISTER studies [16], patients had to be in MR5 and MR4.5, respectively, to enroll on those studies. Later, several studies demonstrated that attempting TFR at

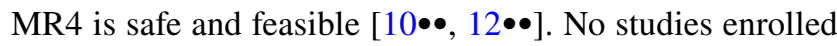
patients who were in accelerated or blast phase, and a TFR attempt in these patients is not currently recommended.

\section{Predictors of Response}

There are several patient-, disease-, and treatment-related characteristics that can predict successful TFR. Older age in two studies was associated with better chance of successful TFR $[17,18]$ (Table 1). For example, in the study by Mori et al., the risk of relapse was $95 \%, 42 \%$, and $33 \%$ for patients $<45,46-64$, and $>64$. However, this finding was not replicated in other studies. Disease-related characteristics affecting TFR outcome include Sokal risk at presentation, prior history of resistance, depth of response, rate of BCR-ABL decline, and BCR-ABL transcript type. A lower Sokal risk is associated with a higher chance of achieving MR4 or MR4.5 and may be associated with a higher chance of achieving TFR. Practically, very few physicians calculate a Sokal risk so this parameter tends not to be useful in everyday practice. In the DASfree study, of the 84 patients enrolled, 25 were resistant to prior therapy [17]. At 2 years, the rate of successful
Table 1 Predictors of treatmentfree remission on multivariate analysis in select studies

\begin{tabular}{|c|c|}
\hline Study & Factors \\
\hline DASfree [17] & $\begin{array}{l}\text { Older age } \\
\text { First-line therapy } \\
\text { Longer treatment duration }\end{array}$ \\
\hline ENESTop [43] & Longer MR4.5 duration prior to discontinuation \\
\hline LAST $[10 \bullet \bullet]$ & $\begin{array}{l}\text { Undetectable BCR-ABL RQ-PCR at } 3 \text { months after discontinuation } \\
\text { Undetectable BCR-ABL1 by digital PCR at time of discontinuation }\end{array}$ \\
\hline DESTINY [20•] & $\begin{array}{l}\text { Longer treatment duration } \\
\text { MR4 group (all BCR-ABL1 measurements after dose reduction }<0.01 \% \text { ) }\end{array}$ \\
\hline 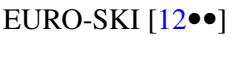 & $\begin{array}{l}\text { Longer treatment duration } \\
\text { Longer MR4 duration prior to discontinuation }\end{array}$ \\
\hline STIM [44] & $\begin{array}{l}\text { Low-risk Sokal } \\
\text { Longer treatment duration }\end{array}$ \\
\hline ISAV [18] & $\begin{array}{l}\text { Older age } \\
\text { Undetectable BCR-ABL1 by digital PCR at time of discontinuation }\end{array}$ \\
\hline
\end{tabular}


TFR was $51 \%$ vs. $44 \%$ in patients on first-line dasatinib vs. those with resistant disease. On multivariate analysis, longer duration of prior dasatinib, older age, and first line of therapy were statistically significantly associated with better chance of maintaining TFR after stopping dasatinib. In the ENESTop study, patients resistant or intolerant to imatinib were switched to nilotinib [19]. Patients who achieved MR4.5 and maintained that response for at least 2 years were eligible to attempt TFR. Of the 126 patients who were eligible to stop therapy, $58 \%$ stayed off drug at 48 weeks. There was no difference between patients who switched for intolerance, resistance, or physician preference. Both of those studies demonstrate the safety and feasibility of attempting TFR for patients with a sustained DMR on second-line therapy. Patients with a deeper response have a better chance of achieving TFR. In the initial studies, only patients with MR5 or undetectable disease were enrolled in TFR studies. Later studies such as EURO-SKI [12••] and Life After Stopping TKI (LAST) [10••] study enrolled patients who were in a sustained MR4. In the EURO-SKI trial, there was no difference in successful TFR rate between patients who were in MR4 vs. MR4.5 or deeper. The DESTINY trial enrolled patients who were at least in MMR [20•]. In those patients, the dose of the TKI was halved for 1 year and patients who maintained MR3 or better attempted TFR. Patients with at least one IS measurement between 0.1 and 0.01 were labeled as the MMR group, while others were labeled as the MR4 group. The rate of successful TFR was $36 \%$ for the MMR group and $72 \%$ for the MR4 group.

Droplet digital PCR is a highly sensitive method for detecting the $B C R-A B L 1$ transcript [21]. Patients with undetectable $B C R-A B L 1$ by ddPCR at time of discontinuation are more likely to achieve a successful TFR than those whose $B C R-A B L 1$ is undetectable by RQ-PCR but detected by ddPCR $[10 \bullet \bullet, 18]$. In the LAST study, the rate of molecular recurrence was higher for patients with detectable $B C R-A B L 1$ by RQ-PCR (50\%) or ddPCR only (64\%) vs. patients with undetectable $B C R-A B L 1$ by both RQ-PCR and ddpCR $(10.3 \%)$ [10••]. In addition, two large studies have demonstrated that the $B C R-A B L 1$ level at 3-month

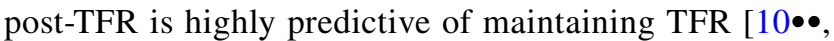
22]. In the ENESTfreedom study, the rate of successful TFR at 96 weeks was $82.6 \%$, vs. $0 \%$ for patients with $B C R$ $A B L 1<0.0032 \%$ vs. those with $B C R-A B L 10.01$ to $0.1 \%$. Transcript type has also been shown to affect rates of successful TFR. Patients with e14a2 BCR-ABL1 transcript type are more likely to achieve MR4.5 and more likely to have a successful TFR after stopping TKIs [23-25]. In a retrospective study from the Adelaide group, $70 \%$ of patients with e 14a $2 B C R-A B L 1$ transcript achieved MR 4.5 by 6 years compared to $52 \%$ with e13a2. Of the patients who were eligible to attempt TFR, patients with e14a2 were more likely to remain in TFR compared to those with e13a2 (65\% vs. 34\%) [23]. Early $B C R-A B L 1$ kinetics predict successful TFR. In one study, patients with $B C R$ - $A B L 1$ halving time $<9.35$ days were more likely to achieve a sustained TFR after discontinuation ( $80 \%$ vs. $4 \%$ ) [26].

The rate of successful TFR is approximately $50 \%$ regardless of type of TKI [3-5]. However, patients who start a second-generation TKI have approximately a $20 \%$ chance higher of achieving MR4. With TFR as the goal of therapy, some may recommend a second-generation TKI in order to get more patients to TFR. Of a theoretical 100 patients starting imatinib, $40 \%$ would achieve MR 4 and $20 \%$ would achieve a successful TFR. Of 100 patients starting a secondgeneration TKI, $60 \%$ would achieve MR4 and 30\% would have a successful TFR. This has never been compared headto-head and remains a theoretical possibility.

\section{Monitoring After TKI Discontinuation}

Although TKI discontinuation clinical trials have not had standardized criteria stating when to restart treatment, it has now become generally accepted by both the NCCN and the ELN that in clinical practice the trigger for re-initiation of TKI therapy in a patient who has stopped treatment is the loss of major molecular response (MMR; $B C R-A B L 1$ transcripts $\geq 0.1 \%$ IS) $[13,14]$.

A meta-analysis compiled data from 3105 patients with reported data on TKI discontinuation. Taking all of the data together, the authors found that the probability of a molecular recurrence within the first 6 months after TKI cessation is $35 \%$. The likelihood of relapse decreases significantly after month 6 , where they found the probability of recurrence in months $7-12$ was $8 \%$, in months $13-18$ was $3 \%$, and in months 19-24 was 3\% [27]. A single-center study looked at 128 patients enrolled on discontinuation trials over a 15 -year period to analyze the rate of late molecular recurrence. Late molecular recurrence was defined as relapse after 2 years in TFR. They found that $14 \%$ of molecular recurrences occur after 2 years. However, when looking at the BCR-ABL1 RQ-PCR values during the TFR period, the incidence of late molecular recurrence in patients whose BCR-ABL1 was consistently $<0.0032 \%$ IS (MR4.5) was $0 \%$, whereas the incidence of late molecular recurrence was approximately $35 \%$ in patients with PCR values that fluctuated between $\geq 0.0032 \%$ and $<0.1 \%$ IS. These data suggest that, although uncommon, late molecular recurrence can occur in patients with fluctuating levels of $B C R-A B L 1$ transcripts in TFR [28].

The NCCN and ELN recommendations for monitoring $B C R-A B L 1$ transcript levels after TKI discontinuation are based on the above data suggesting that the highest risk time period is in the first 6 months after stopping therapy. 
Although the possibility of molecular recurrence does not ever become zero, the likelihood of remaining in TFR raises after the first 6-12 months. For this reason, both sets of guidelines suggest monitoring $B C R-A B L 1$ transcript levels by RQ-PCR monthly for the first 6 months after stopping treatment, and then every 2 months between months 7 and 12. In those patients who remain in TFR at 12 months, the frequency of PCR monitoring can be decreased to every 3 months at that time point. Given the potential for late recurrence, it is never recommended to stop monitoring altogether [13, 14].

\section{Side Effects of Discontinuation}

Side effects associated with discontinuation include a withdrawal syndrome, anxiety, and increased visits to healthcare settings. TKI withdrawal syndrome occurs in approximately $30 \%$ of patients [29-31]. It is characterized by muscle and joint pain after discontinuation. It generally begins days to weeks after discontinuation and lasts for approximately 6 months. Treatment is supportive care with NSAIDs; acetaminophen and rarely steroids may be used. Restarting TKIs may be required in a very small number of patients who require long-term steroids or narcotics. The mechanism for the musculoskeletal pain remains unclear although several theories including release of c-Kit inhibition [32], mast cell activation [33], and bone remodeling effects [34] have been suggested. Another side effect is increased anxiety after discontinuation. Patients express increased anxiety at the time of each lab check, waiting to see if they will need to restart their medications. Lastly, although not truly a side effect, increased visits to the healthcare setting is a significant concern associated with TKI discontinuation. This was highlighted during the COVID-19 pandemic where some patients elected to wait to discontinue TKI until after the pandemic in order to decrease their visits to healthcare facilities.

\section{Quality of Life After Discontinuation}

A primary motivator for TKI cessation is the idea that without daily treatment that has the potential to cause immediate- and long-term toxicity, patient-reported quality of life will improve. The first TFR study to objectively assess patient-reported outcomes (PROs) was the Life After Stopping TKIs (LAST) study. On this trial, PROs were assessed every month during the first 6 months on the trial, then again at months 8 and 12 , and then every 6 months until the completion of 36 months. In those patients with a molecular recurrence who restarted TKI therapy, PROs were assessed quarterly for the first year after re-initiation of treatment, and then every 6 months.
The objectives were to describe patient-reported symptoms after TKI discontinuation and after restarting TKI therapy. After analyzing 1883 PRO assessments, investigators on this trial found that TKI discontinuation was associated with a statistically significant improvement in fatigue, depression, diarrhea, and sleep disturbance. These worsened in patients who restarted treatment at the time of molecular recurrence. Interestingly, even in those patients with symptoms consistent with TKI withdrawal syndrome, this was rarely reported to interfere with daily life $[10 \bullet \bullet]$.

Another study which reported on patient perspectives surrounding TFR found that $31 \%$ of patients felt fearful or anxious just prior to or immediately after getting their PCR monitoring completed; however, a larger percentage of patients (44\%) stated they never felt fearful or anxious at all. This study concluded that in many cases, the patients' psychological well-being is not adequately addressed by the treating physician leaving patients to feel uneasy about TKI cessation [7].

In general, the favorable impact on patient-reported outcomes and health-related quality of life, support attempting TKI cessation in eligible patients. A thorough discussion of the potential risks, benefits, and side effects, including TKI withdrawal syndrome should be done, prior to attempting discontinuation.

\section{Second Treatment-Free Remission}

Currently, the NCCN and ELN guidelines recommend restarting TKI therapy at the time of molecular recurrence after the first TFR attempt with the intent of remaining on lifelong treatment. Although second TFR is not considered standard practice, there have been small cohorts of patients who have attempted this. A meta-analysis of all published data on TFR identified only 124 recorded patients attempting a second TFR [27]. The largest dataset comes from the 70 patients enrolled on the French RE-STIM study in which $35 \%$ of patients remained in TFR2 at 36 months [35]. It is important to note that the trigger for restarting treatment after the first TFR attempt in patients on this trial was loss of MR4.5 rather than loss of MMR, and it is unclear how this may have impacted the outcomes of the RE-STIM trial. Twelve patients enrolled on the Australian CML8 Study discontinued TKIs after regaining a deep molecular response following the first TFR attempt. In this case, $50 \%$ of the patients remain in TFR 2 with a median follow-up of 8.6 years [36].

At this time, it is too early to consider incorporating second TFR into standard practice given the paucity of available data. However, the limited data published suggests that TFR2 is safe and feasible in a subset of patients. 


\section{Conclusions and Future Directions}

Treatment-free remission should be considered the ultimate goal for patients with CML. Several ongoing studies aim to increase the proportion of patients who can successfully achieve TFR. Studies adding ruxolitinib or pembrolizumab to TKI therapy for patients who have not achieved MR4.5 are ongoing. Other studies are evaluating the role of adding ruxolitinib or asciminib to TKI therapy in patients who were not able to achieve a sustained TFR after a first attempt of TKI discontinuation [37]. In addition, a better understanding of why some patients with detectable $B C R-A B L 1$ level do not develop clinical disease is an area of future investigation [38]. Several theories explaining this phenomena have been proposed including that the BCR-ABL1 is in memory lymphocytes not the myeloid lineage [39•], leukemia stem cell exhaustion associated with shorter telomeres [40], or an immune surveillance effect as patients with a higher T [41] or NK cell [42] numbers have a higher likelihood of achieving TFR. Ultimately, our goal is maintain the excellent survival achieved by TKIs, and improve patients quality of life. Improving quality of life could be achieved by successful TFR, lowering the TKI dose or optimizing supportive care.

\section{Declarations}

Human and Animal Rights and Informed Consent This article does not contain any studies with human or animal subjects performed by any of the authors.

\section{References}

Papers of particular interest, published recently, have been highlighted as:

- Of importance

•• Of major importance

1. Deininger MW, Goldman JM, Melo JV. The molecular biology of chronic myeloid leukemia. Blood. 2000;96(10):3343-56.

2. O'Brien SG, Fo Guilhot, Larson RA, Gathmann I, Baccarani M, Cervantes F, et al. Imatinib compared with interferon and lowdose cytarabine for newly diagnosed chronic-phase chronic myeloid leukemia. New England J Med. 2003;348(11):994-1004. https://doi.org/10.1056/NEJMoa022457.

3. Cortes JE, Saglio G, Kantarjian HM, Baccarani M, Mayer J, Boqué C, et al. Final 5-year study results of DASISION: the dasatinib versus imatinib study in treatment-naïve chronic myeloid leukemia patients trial. J Clin Oncol. 2016;34(20):2333-40. https://doi.org/10.1200/jco.2015.64.8899.

4. Hochhaus A, Saglio G, Hughes TP, Larson RA, Kim DW, Issaragrisil S, et al. Long-term benefits and risks of frontline nilotinib vs imatinib for chronic myeloid leukemia in chronic phase: 5 -year update of the randomized ENESTnd trial. Leukemia. 2016;30(5):1044-54. https://doi.org/10.1038/leu.2016.5.
5. Brümmendorf TH, Cortes JE, Milojkovic D, Gambacorti-Passerini C, Clark RE, le Coutre PD, et al. Bosutinib (BOS) versus imatinib for newly diagnosed chronic phase (CP) chronic myeloid leukemia (CML): final 5-year results from the Bfore trial. Blood. 2020;136(Supplement 1):41-2. https://doi.org/ 10.1182/blood-2020-137393.

6. Kantarjian HM, Deininger MW, Abruzzese E, Apperley J, Cortes JE, Chuah C, et al. Efficacy and safety of ponatinib (PON) in patients with chronic-phase chronic myeloid leukemia (CPCML) who failed one or more second-generation (2G) tyrosine kinase inhibitors (TKIs): analyses based on PACE and Optic. Blood. 2020;136(Supplement 1):43-4. https://doi.org/10.1182/ blood-2020-133922.

7. Sharf G, Marin C, Bradley JA, Pemberton-Whiteley Z, Bombaci F, Christensen RIO, et al. Treatment-free remission in chronic myeloid leukemia: the patient perspective and areas of unmet needs. Leukemia. 2020;34(8):2102-12. https://doi. org/10.1038/s41375-020-0867-0.

8. Goldberg S, Hamarman S. Patients with chronic myelogenous leukemia may not want to discontinue tyrosine kinase inhibitor therapy. Blood. 2015;126(23):1584.

9. Flynn KE, Myers JM, D’Souza A, Schiffer CA, Thompson JE, Atallah E. Exploring patient decision making regarding discontinuation of tyrosine kinase inhibitors for chronic myeloid leukemia. Oncologist. 2019;24(9):1253-8. https://doi.org/10. 1634/theoncologist.2018-0831.

10.• Atallah E, Schiffer CA, Radich JP, Weinfurt KP, Zhang MJ, Pinilla-Ibarz J et al. Assessment of outcomes after stopping tyrosine kinase inhibitors among patients with chronic myeloid leukemia: a nonrandomized clinical trial. JAMA Oncol. 2020https://doi.org/10.1001/jamaoncol.2020.5774. Large US study evaluating treatment free remission in patients receiving different TKIs. Study included a comprehensive patients reported outcome component

11. Cirmi S, El Abd A, Letinier L, Navarra M, Salvo F. Cardiovascular toxicity of tyrosine kinase inhibitors used in chronic myeloid leukemia: an analysis of the FDA Adverse Event Reporting System Database (FAERS). Cancers (Basel). 2020;12(4). doi:https://doi.org/10.3390/cancers12040826.

12.• Saussele S, Richter J, Guilhot J, Gruber FX, Hjorth-Hansen H, Almeida A, et al. Discontinuation of tyrosine kinase inhibitor therapy in chronic myeloid leukaemia (EURO-SKI): a prespecified interim analysis of a prospective, multicentre, nonrandomised, trial. Lancet Oncol. 2018. https://doi.org/10.1016/ S1470-2045(18)30192-X. Largest European study evaluating treatment free remission.

13. Hochhaus A, Baccarani M, Silver RT, Schiffer C, Apperley JF, Cervantes F, et al. European LeukemiaNet 2020 recommendations for treating chronic myeloid leukemia. Leukemia. 2020;34(4):966-84. https://doi.org/10.1038/ s41375-020-0776-2.

14. Network NCC. Chronic Myeloid Leukemia (Version 3.2021). 2021. https://www.nccn.org/professionals/physician_gls/pdf/ cml.pdf. Accessed July3, 2021.

15. Mahon FX, Rea D, Guilhot J, Guilhot F, Huguet F, Nicolini $\mathrm{F}$, et al. Discontinuation of imatinib in patients with chronic myeloid leukaemia who have maintained complete molecular remission for at least 2 years: the prospective, multicentre Stop Imatinib (STIM) trial. Lancet Oncol. 2010;11(11):1029-35. https://doi.org/10.1016/S1470-2045(10)70233-3.

16. Ross DM, Branford S, Seymour JF, Schwarer AP, Arthur C, Yeung DT, et al. Safety and efficacy of imatinib cessation for CML patients with stable undetectable minimal residual disease: results from the TWISTER study. Blood. 2013;122(4):515-22. https://doi.org/10.1182/blood-2013-02-483750. 
17. Shah NP, García-Gutiérrez V, Jiménez-Velasco A, Larson S, Saussele S, Rea D, et al. Dasatinib discontinuation in patients with chronic-phase chronic myeloid leukemia and stable deep molecular response: the DASFREE study. Leuk Lymphoma. 2020;61(3):650-9. https://doi.org/10.1080/10428194.2019. 1675879.

18. Mori S, Vagge E, le Coutre P, Abruzzese E, Martino B, Pungolino E, et al. Age and dPCR can predict relapse in CML patients who discontinued imatinib: the ISAV study. Am J Hematol. 2015;90(10):910-4. https://doi.org/10.1002/ajh. 24120.

19 Hughes TP, Boquimpani CM, Takahashi N, Benyamini N, Clementino NCD, Shuvaev V, et al. Treatment-free remission in patients with chronic myeloid leukemia in chronic phase according to reasons for switching from imatinib to nilotinib: subgroup analysis from ENESTop. Blood. 2016;128(22):792. https://doi. org/10.1182/blood.V128.22.792.792.

20. Clark RE, Polydoros F, Apperley JF, Milojkovic D, Rothwell $\mathrm{K}$, Pocock C, et al. De-escalation of tyrosine kinase inhibitor therapy before complete treatment discontinuation in patients with chronic myeloid leukaemia (DESTINY): a non-randomised, phase 2 trial. Lancet Haematol. 2019;6(7):e375-83. https://doi. org/10.1016/S2352-3026(19)30094-8. This study used a different approach to discontinuation. The TKI dose was first reduced for one year. In patients who mainatined MMR, the TKI was then stopped.

21. Jennings LJ, George D, Czech J, Yu M, Joseph L. Detection and quantification of BCR-ABL1 fusion transcripts by droplet digital PCR. J Mol Diagn. 2014;16(2):174-9. https://doi.org/10.1016/j. jmoldx.2013.10.007.

22. Ross DM, Masszi T, Gómez Casares MT, Hellmann A, Stentoft $\mathrm{J}$, Conneally E, et al. Durable treatment-free remission in patients with chronic myeloid leukemia in chronic phase following frontline nilotinib: 96-week update of the ENESTfreedom study. J Cancer Res Clin Oncol. 2018;144(5):945-54. https:// doi.org/10.1007/s00432-018-2604-x.

23 Shanmuganathan N, Branford S, Yong ASM, Hiwase DK, Yeung DT, Ross DM, et al. The e13a2 BCR-ABL1 transcript is associated with higher rates of molecular recurrence after treatmentfree remission attempts: retrospective analysis of the Adelaide cohort. Blood. 2018;132(Supplement 1):1731. https://doi.org/ 10.1182/blood-2018-99-111083.

24. Baccarani M, Rosti G, Soverini S. Chronic myeloid leukemia: the concepts of resistance and persistence and the relationship with the BCR-ABL1 transcript type. Leukemia. 2019;33(10):235864. https://doi.org/10.1038/s41375-019-0562-1.

25. D'Adda M, Farina M, Schieppati F, Borlenghi E, Bottelli C, Cerqui E, et al. The e13a2 BCR-ABL transcript negatively affects sustained deep molecular response and the achievement of treatment-free remission in patients with chronic myeloid leukemia who receive tyrosine kinase inhibitors. Cancer. 2019;125(10):1674-82. https://doi.org/10.1002/cncr.31977.

26. Shanmuganathan N, Pagani IS, Ross DM, Park S, Yong ASM, Braley JA, et al. Early BCR-ABL1 kinetics are predictive of subsequent achievement of treatment-free remission in chronic myeloid leukemia. Blood. 2021;137(9):1196-207. https://doi. org/10.1182/blood.2020005514.

27. Dulucq S, Astrugue C, Etienne G, Mahon FX, Benard A. Risk of molecular recurrence after tyrosine kinase inhibitor discontinuation in chronic myeloid leukaemia patients: a systematic review of literature with a meta-analysis of studies over the last ten years. Br J Haematol. 2020;189(3):452-68. https://doi.org/ 10.1111/bjh.16408.

28. Rousselot P, Loiseau C, Delord M, Cayuela JM, Spentchian M. Late molecular recurrences in patients with chronic myeloid leukemia experiencing treatment-free remission. Blood Adv.
2020;4(13):3034-40. https://doi.org/10.1182/bloodadvances. 2020001772.

29. Diab M, Schiffer CA. The spectrum of musculoskeletal symptoms in patients with chronic myeloid leukemia after stopping tyrosine kinase inhibitors. Leuk Res. 2019;79:1-2. https://doi. org/10.1016/j.leukres.2019.02.001.

30. Kota V, Atallah E. Musculoskeletal pain in patients with chronic myeloid leukemia after tyrosine kinase inhibitor therapy cessation. Clin Lymphoma Myeloma Leuk. 2019;19(8):480-7. https:// doi.org/10.1016/j.clml.2019.05.007.

31. Richter J, Soderlund S, Lubking A, Dreimane A, Lotfi K, Markevarn B, et al. Musculoskeletal pain in patients with chronic myeloid leukemia after discontinuation of imatinib: a tyrosine kinase inhibitor withdrawal syndrome? J Clin Oncol. 2014;32(25):2821-3. https://doi.org/10.1200/JCO.2014.55. 6910 .

32. Ceko M, Milenkovic N, le Coutre P, Westermann J, Lewin GR. Inhibition of c-Kit signaling is associated with reduced heat and cold pain sensitivity in humans. PAIN@. 2014;155(7):1222-8. https://doi.org/10.1016/j.pain.2014.03.010.

33. Ramanujam D, McNicholl F, Furby D, Richardson D, Cuthbert RJG, McMullin MF. Dramatic resolution of respiratory symptoms with imatinib mesylate in patients with chronic myeloid leukemia presenting with lower airway symptoms resembling asthma. Leuk Lymphoma. 2009;50(10):1721-2. https://doi.org/ 10.1080/10428190903161059.

34. Breccia M, Alimena $\mathrm{G}$. The metabolic consequences of imatinib mesylate: changes on glucose, lypidic and bone metabolism. Leuk Res. 2009;33(7):871-5. https://doi.org/10.1016/j.leukres. 2009.01.040.

35. Legros L, Nicolini FE, Etienne G, Rousselot P, Rea D, Giraudier $\mathrm{S}$, et al. Second tyrosine kinase inhibitor discontinuation attempt in patients with chronic myeloid leukemia. Cancer. 2017;123(22):4403-10. https://doi.org/10.1002/cncr.30885.

36. Ross DM, Pagani IS, Shanmuganathan N, Kok CH, Seymour JF, Mills AK, et al. Long-term treatment-free remission of chronic myeloid leukemia with falling levels of residual leukemic cells. Leukemia. 2018;32(12):2572-9. https://doi.org/10.1038/ s41375-018-0264-0.

37. Clinicaltrials.gov. 2017. https://www.clinicaltrials.gov/ct2/ show $/$ NCT03610971 cond $=\mathrm{cml}+$ ruxolitinib \& draw $=2 \&$ rank=4, https://www.clinicaltrials.gov/ct2/show/NCT04838041? cond $=\mathrm{cml}+$ asciminib \&draw $=2 \&$ rank $=7$.

38. Ross DM, Branford S, Seymour JF, Schwarer AP, Arthur C, Bartley PA, et al. Patients with chronic myeloid leukemia who maintain a complete molecular response after stopping imatinib treatment have evidence of persistent leukemia by DNA PCR. Leukemia. 2010;24(10):1719-24. https://doi.org/10.1038/leu. 2010.185.

39. Pagani IS, Dang P, Saunders VA, Grose R, Shanmuganathan $\mathrm{N}$, Kok $\mathrm{CH}$, et al. Lineage of measurable residual disease in patients with chronic myeloid leukemia in treatment-free remission. Leukemia. 2020;34(4):1052-61. https://doi.org/10.1038/ s41375-019-0647-x. Study suggesting that BCR-ABL1 is not detectable in the myeloid lineage in patients who maintain a successful TFR.

40. Caocci G, Greco M, Delogu G, Secchi C, Martino B, Labate $\mathrm{C}$, et al. Telomere length shortening is associated with treatment-free remission in chronic myeloid leukemia patients. J Hematol Oncol. 2016;9(1):63. https://doi.org/10.1186/ s13045-016-0293-y.

41. Cayssials E, Jacomet F, Piccirilli N, Lefèvre L, Roy L, Guilhot $\mathrm{F}$, et al. Sustained treatment-free remission in chronic myeloid leukaemia is associated with an increased frequency of innate CD8(+) T-cells. Br J Haematol. 2019;186(1):54-9. https://doi. org/10.1111/bjh.15858. 
42. Ilander M, Olsson-Stromberg U, Schlums H, Guilhot J, Bruck O, Lahteenmaki $\mathrm{H}$, et al. Increased proportion of mature NK cells is associated with successful imatinib discontinuation in chronic myeloid leukemia. Leukemia. 2017;31(5):1108-16. https://doi. org/10.1038/leu.2016.360.

43. Hughes TP, Clementino NCD, Fominykh M, Lipton JH, Turkina AG, Moiraghi EB, et al. Long-term treatment-free remission in patients with chronic myeloid leukemia after second-line nilotinib: ENESTop 5-year update. Leukemia. 2021;35(6):1631-42. https://doi.org/10.1038/s41375-021-01260-y.
44. Etienne G, Guilhot J, Rea D, Rigal-Huguet F, Nicolini F, Charbonnier A, et al. Long-Term Follow-Up of the French Stop Imatinib (STIM1) Study in Patients With Chronic Myeloid Leukemia. J Clin Oncol. 2017;35(3):298-305. https://doi.org/ 10.1200/JCO.2016.68.2914.

Publisher's Note Springer Nature remains neutral with regard to jurisdictional claims in published maps and institutional affiliations. 